\title{
ON SEMI-GROUPS OF UNBOUNDED NORMAL OPERATORS
}

\section{R. K. GETOOR ${ }^{1}$}

In this note we discuss the integral representation of a semi-group of unbounded normal operators. The result for a semi-group of bounded normal operators can be found in Sz. Nagy [4]. Recently A. Devinatz [1] obtained a similar result for semi-groups of unbounded self-adjoint operators. The following theorem is proved.

TheORem. Let $\left\{N_{t} ; t>0\right]$ be a semi-group (i.e., $N_{t} N_{s}=N_{t+s}$ ) of normal operators on a Hilbert space 3 C. Let $D_{t}$ be the domain of $N_{t}$ (each $D_{t}$ dense in $\mathfrak{H C}$ ) and let $D=\bigcap_{t>0} D_{t}$ then we suppose that $N_{t} x$ is weakly continuous as a function of $t(t>0)$ for each fixed $x \in D$. Then there exists a unique complex spectral resolution $K(\Lambda)$ whose support is contained in $\lambda_{1} \geqq 0, \lambda=\lambda_{1}+i \lambda_{2}$, such that

$$
N_{t}=\int_{\lambda_{1} \geqq 0} \lambda_{1}^{t} e^{i \lambda_{2} t} K(d \lambda), \quad t>0 .
$$

Proof. First recall that if $N$ is a normal operator then $N=A U$ $=U A$ where $U$ is unitary and $A$ is self-adjoint and has the same domain as $N$. In fact if $K(\Lambda)$ is the spectral resolution of $N$ then we can define $A$ and $U$ as follows,

$$
\begin{aligned}
A & =\int|\lambda| K(d \lambda), \\
U & =\int s(\lambda) K(d \lambda) \text { where } s(\lambda)=\left\{\begin{array}{cc}
\lambda /|\lambda|, & \lambda \neq 0, \\
1, & \lambda=0 .
\end{array}\right.
\end{aligned}
$$

Let us also note that if $p$ is an integer then $\left(N^{p}\right)^{*}=\left(A^{p} U^{p}\right)^{*}=A^{p} U^{-p}$ and that $\left(N^{*}\right)^{p}=\left(A U^{*}\right)^{p}=A^{p} U^{-p}$, hence $\left(N^{p}\right)^{*}=\left(N^{*}\right)^{p}$.

We now prove a series of simple statements which taken together yield our theorem.

(a) If $t$ and $s$ are commensurable then $N_{t} N_{s}^{*}=N_{s}^{*} N_{t}$. This is a trivial consequence of the semi-group property of $\left\{N_{t}, t>0\right\}$ and the fact that $\left(N_{t}^{*}\right)^{n}=\left(N_{t}^{n}\right)^{*}$.

Presented to the Society, December 27, 1954; received by the editors June 3, 1955.

${ }^{1}$ This research was supported in part by the Office of Ordnance Research contract DA-36-034-ORD-1296RD. 
(b) If $x \in D$ then $N_{t} N_{s}^{*} x=N_{s}^{*} N_{t} x$ for all $t, s>0$. First if $x \in D$ then $N_{t} x \in D$ and $N_{t}^{*} x \in D$ for all $t>0$, since if $x \in D$ then $N_{t+8} x$ exists and $N_{s} N_{t} x=N_{t+8} x$. Thus $N_{t} x \in D_{s}$ for all $s$ and hence is in $D$. To show $N_{t}^{*} x \in D$, let $t_{0}>0$ be fixed, then $N_{s} x \in D$ for all $s>0$ as seen above, hence $N_{t_{0}}^{*} N_{\varepsilon} x$ exists. (Domain of $N_{t_{0}}^{*}$ is $D_{t_{0}}$ since $N_{t_{0}}$ is normal.) Let $s=n t_{0}, n=1,2, \cdots$, then since $s$ and $t_{\theta}$ are commensurable $N_{t_{0}}^{*} N_{s} x=N_{s}\left(N_{t_{0}}^{*} x\right)$ or $N_{t_{0}}^{*} x \in D_{n t_{0}}$ for $n=1,2 \cdots$ But by the semi-group property of $N_{t}, t>0$, the $D_{t}$ 's are a decreasing collection of sets and therefore $D=\bigcap_{t>0} D_{t}=\lim _{t \rightarrow \infty} D_{t}=\lim _{n \rightarrow \infty} D_{n t_{0}}$ since $t_{0}>0$. Thus $N_{t_{0}}^{*} x \in D$.

If $t, s$ are given and $x \in D$ then $N_{t} N_{s}^{*} x$ and $N_{s}^{*} N_{t} x$ both exist. Let $t_{n} \rightarrow t, t_{n}$ and $s$ commensurable. A standard argument making use of the continuity property of $\left\{N_{t}, t>0\right\}$ shows that for a fixed $x \in D\left(N_{s}^{*} N_{t} x, y\right)=\left(N_{t} N_{s}^{*} x, y\right)$ for all $y \in D_{s}$, but $D_{s}$ is dense in $\mathcal{F}$ and hence $N_{s}^{*} N_{t} x=N_{t} N_{s}^{*} x$.

(c) If we define $A_{t}=N_{t / 2}^{*} N_{t / 2}, t>0$, then $\left\{A_{t}, t>0\right\}$ is a semigroup of self-adjoint operators such that

$$
A_{t}=A_{1}^{t}=\int_{0}^{\infty} \lambda_{1}^{t} d E\left(\lambda_{1}\right), \quad t>0 .
$$

It is clear from the definition that each $A_{t}$ is a positive self-adjoint operator and moreover $A_{t}^{2}=N_{t}^{*} N_{t}$. Since a positive self-adjoint operator has a unique positive self-adjoint square root it follows that $A_{t}$ is the operator defined by (2) for $N_{t}$. Thus $D_{A_{t}}=D_{t}$ and $D=\bigcap_{t>0} D_{A_{t}}$.

If $x \in D$ then $A_{t} A_{s} x=N_{t / 2}^{*} N_{t / 2} N_{s / 2}^{*} N_{s / 2} x$ and this is defined since $N_{t} D \subset D$ and $N_{t}^{*} D \subset D$ for all $t>0$. Moreover by (b)

$$
A_{t} A_{s} x=N_{t / 2}^{*} N_{s / 2}^{*} N_{t / 2} N_{s / 2} x=N_{(t+s) / 2}^{*} N_{(t+s) / 2} x=A_{t+8} x,
$$

since in general $N_{(t+s) / 2}^{*} \supset N_{t / 2}^{*} N_{s / 2}^{*}$. Also

$$
\begin{aligned}
A_{t} A_{8} x & =N_{s / 2}^{*} N_{t / 2}^{*} N_{s / 2} N_{t / 2} x \\
& =N_{s / 2}^{*} N_{8 / 2} N_{t / 2}^{*} N_{t / 2} x=A_{8} A_{t} x .
\end{aligned}
$$

Consequently if $x \in D, A_{t} A_{s} x=A_{s} A_{t} x=A_{s+t} x$. Now the same argument as used by Sz. Nagy [4, p. 74] shows that $\left(A_{t} x, x\right)$ for each fixed $x \in D$ is bounded above as a function of $t$ in every interval $0<a \leqq t \leqq b$. This implies, Sz. Nagy $\left[4\right.$, p. 73], that $\left(A_{t} x, x\right)$ is continuous for $t>0$ and $x \in D$. We would like to apply Devinatz's theorem at this point to the $A_{t}$ 's but we do not know a priori that the $A_{t}$ 's form a semi-group. The following argument is almost word for word that of Devinatz $\left[1\right.$, p. 102]. Define $H_{t}=A_{1}^{t}$. Clearly $\left\{H_{t} ; t>0\right\}$ 
is a semi-group. In addition using (a) we see that $H_{n}=A_{1}^{n}=\left(N_{1 / 2}^{*} N_{1 / 2}\right)^{n}$ $=N_{n / 2}^{*} N_{n / 2}=A_{n}$. Furthermore the uniqueness of square roots of positive self-adjoint operators implies that for any integers $n, m, H_{n / 2^{m}}$ $=A_{n / 2^{m}}$. Now, there exists a countable set of mutually orthogonal manifolds $\left\{M_{k}\right\}_{1}^{\infty}$, whose direct sum is the whole space and such that, for all $t>0, H_{t}=\sum_{k=1}^{\infty} \oplus H_{t}^{(k)}$, where $H_{t}^{(k)}$ is a bounded selfadjoint operator on $M_{k}$ and is the restriction of $H_{t}$ to $M_{k}$ (Sz. Nagy $\left[4\right.$, p. 49]). That is, $x \in D_{H_{t}}$ if and only if $\sum_{k=1}^{\infty}\left\|H_{t}^{(k)} x_{k}\right\|^{2}<\infty$, where $x_{k} \in M_{k}$, and $x=\sum_{k=1}^{\infty} x_{k}$. Then $H_{t} x=\sum_{k=1}^{\infty} H_{t}^{(k)} x_{k}$.

Given any $t>0$ there exists an $m / 2^{n} \geqq t$. From thesemi-group property of $\left\{N_{t}, t>0\right\}$ we know that $D_{m / 2}{ }^{n} \subset D_{t}\left(D_{t}\right.$ is also the domain of $\left.A_{t}\right)$. Thus for every $x_{k} \in M_{k}, x_{k} \in D_{m / 2^{n}} \subset D_{t}$. Consequently, since $H_{m / 2^{n}}$ $=A_{m / 2^{n}}$ and by the continuity of $\left(A_{t} x_{k}, x_{k}\right)$ and $\left(H_{t} x_{k}, x_{k}\right)$ as functions of $t$, we must have $A_{t} x_{k}=H_{t}^{(k)} x_{k}=H_{t} x_{k}$. This implies that $H_{t}=A_{t}$ (Sz. Nagy [4, p. 35]), and hence (4) is proved.

For any $t>0$ the above argument shows that $M_{k} \subset D_{t}$ for $k=1,2, \cdots$, and hence $M_{k} \subset D$ for all $k$. Thus if $x \in \mathcal{F}$ and $x=\sum_{k=1}^{\infty} x_{k}$ then $y_{n}=\sum_{k=1}^{n} x_{k}$ is in $D$ and $y_{n} \rightarrow x$. That is $D$ is dense in $\mathcal{H}$. Moreover for any $t>0$ if $x \in D_{t}$ then $A_{t} y_{n}=\sum_{k=1}^{n} A_{t} x_{k}$ $=\sum_{k=1}^{n} H_{t}^{(k)} x_{k} \rightarrow H_{t} x=A_{t} x$. Thus for any $x \in D_{t}$ there exists a sequence $y_{n} \in D$ such that $y_{n} \rightarrow x$ and $A_{t} y_{n} \rightarrow \mathrm{A}_{t} x$.

For each $t>0$ let $U_{t}$ be the unitary operator defined by (3) such that $N_{t}=A_{t} U_{t}=U_{t} A_{t}$. From (2) and (4) it follows that $A_{t}$ and $N_{s}$ have the same null space, $\Re$, for all $t, s>0 . \Re$ is a closed linear manifold since the operators in question are closed. If we write $\mathfrak{H}=R \oplus \mathfrak{N}$ where $R$ is the orthogonal complement of $\mathfrak{N}$, then $R$ can be characterized as either the closure of $R_{A t}$ or the closure of $R_{N_{t}}$ for any $t>0$. (If $T$ is an operator $R_{T}$ denotes the range of $T$.) Thus $\Re$ is the null space of $A_{t}, N_{t}$, and $N_{t}^{*}$ for all $t>0$ and if we write (3) with the proper subscript we see that $\mathscr{N}$ is also the null space of $U_{t}$ for all $t>0$. (Note that $K_{t}(\{0\})$ is the projection on $\Re$.) It is now clear that all of the above operators are reduced by $\mathfrak{N}$. (A normal operator is always reduced by its null space.) Thus we can restrict all the operators in question to $R$. We assume $R \neq\{0\}$ since in this case everything is trivial.

(d) If $\widetilde{D}=D \cap \Omega$ then $\tilde{D}$ is dense in $R$. Assume $\tilde{D}$ not dense in $R$ then there exists $r \in R, r \neq 0$, such that $r \perp \tilde{D}$. Let $x \in D$, then $x=x_{R}+n$, $x_{R} \in R, n \in \mathcal{H}$. Since $n \in D$ and $D$ is linear we see that $x_{R}=x-n \in D$ and hence in $\tilde{D}$. Therefore $(r, x)=\left(r, x_{R}\right)+(r, n)=0$ which implies that $r=0$ as an element of $\mathfrak{H C}$ since $D$ is dense in $\mathfrak{H C}$. But this contradicts the fact that $r \neq 0$ as an element of $R$.

(e) In this section all operators are considered as operators on $R$. 
If we define $U_{0}=I$ and $U_{-t}=U_{t}^{-1}$ then $\left\{U_{t} ;-\infty<t<\infty\right\}$ is a strongly continuous group of unitary operators on $R$.

First we show that $A_{t} U_{s} x=U_{s} A_{t} x$ for $x \in \tilde{D}$. Note that $x \in \tilde{D}$ implies $A_{t} x \in \widetilde{D}$ by the same argument as that used in (b), and also that (b) and the definition of $A_{t}$ implies $A_{t} N_{s} x=N_{s} A_{t} x$ for all $x \in \tilde{D}$. Thus for all $x \in \tilde{D}$ we have

$$
A_{t} N_{\mathrm{s}} x=N_{\mathrm{s}} A_{t} x, \quad A_{t} A_{\mathrm{s}} U_{\mathrm{s}} x=A_{\mathrm{s}} U_{\mathrm{s}} A_{t} x .
$$

However, the semi-group property of $\left\{A_{t}, t>0\right\}$ implies $A_{t} A_{\mathrm{s}}=A_{\mathrm{s}} A_{t}$, hence

$$
A_{8} A_{t} U_{s} x=A_{8} U_{8} A_{t} x
$$

and from this we conclude that $A_{t} U_{s} x=U_{s} A_{t} x$ since $A_{s}$ is one-to-one in $R$, i.e., $A_{s}^{-1}$ exists. A consequence of this is that $U_{s} x \in \tilde{D}$ if $x \in \tilde{D}$. Therefore for $x \in \tilde{D}, N_{t} N_{s} x=N_{t+8} x=A_{t+s} U_{t+s} x=A_{t} A_{s} U_{t+8} x$ or $U_{t+8} x$ $=A_{8}^{-1} A_{t}^{-1} A_{t} U_{t} A_{8} U_{8} x=U_{t} U_{8} x$. But the $U_{t}^{\prime}$ s are bounded and $\tilde{D}$ is dense in $R$ thus $U_{t} U_{s}=U_{t+8}=U_{s} U_{t}$ and if we define $U_{0}=I$ and $U_{-t}=U_{t}^{*}$ it is clear that $\left\{U_{t} ;-\infty<t<\infty\right\}$ is a group of unitary operators on $R$.

We now investigate the continuity properties of this group. To this end we first note that an immediate consequence of (4) is that $A_{t}$ is strongly continuous on $\tilde{D}$ even at $t=0$, and that $A_{t} x$ is strongly left continuous at $t=t_{0}$ for $x \in \widetilde{D}_{t_{0}}=D_{t_{0}} \cap R$ (if $s \leqq t D_{t} \subset D_{s}$ and hence $\left.\tilde{D}_{t} \subset \widetilde{D}_{\varepsilon}\right)$. Suppose $t_{0}>0$ and $t_{n} \uparrow t_{0}, 0<t_{n}<t_{0}$, then $\widetilde{D}_{t_{0}} \subset \widetilde{D}_{t_{n}}$. Let $x \in R_{A_{t_{0}}}, y \in \widetilde{D}$ then $x=A_{t_{0}} z$ where $z \in \widetilde{D}_{t_{0}} \subset \widetilde{D}_{t_{n}}$. Thus we have

$$
\begin{aligned}
\mid\left(U_{-t_{n}} x, y\right) & -\left(U_{-t_{0}} x, y\right)|=|\left(U_{-t_{n}} A_{t_{0}} z, y\right)-\left(U_{-t_{0}} A_{t_{0}} z, y\right) \mid \\
& \leqq\left|\left(U_{-t_{n}}\left[A_{t_{0}}-A_{t_{n}}\right] z, y\right)\right|+\left|\left(\left[U_{-t_{n}} A_{t_{n}}-U_{-t_{0}} A_{t_{0}}\right] z, y\right)\right| \\
& \leqq\left\|\left(A_{t_{0}}-A_{t_{n}}\right) z\right\| \cdot\|y\|+\left|\left(N_{t_{n}}^{*} z, y\right)-\left(N_{t_{0}}^{*} z, y\right)\right| \\
& =\left\|\left(A_{t_{0}}-A_{t_{n}}\right) z\right\| \cdot\|y\|+\left|\left(z, N_{t_{n}} y\right)-\left(z, N_{t_{0}} y\right)\right| \\
& \rightarrow 0 \text { as } t_{n} \uparrow t_{0} .
\end{aligned}
$$

Since $R_{A_{t_{0}}}$ and $\tilde{D}$ are dense in $R$ and $\left\|U_{t}\right\|=1$ it follows that $\left\{U_{t}, t<0\right\}$ is weakly right continuous. However, weak right continuity at any one point $t_{0}$ implies weak right continuity for all $t$ since $\left(\left[U_{t+h}-U_{t}\right] x, y\right)$ $=\left(\left[U_{t_{0}+h}-U_{t_{0}}\right] x, U_{t_{0}-t} y\right)$. A minor modification in the proof of Theorem 9.2.2 in Hille [3] shows that $U_{t}$ is strongly continuous for all $t$. This completes the proof of (e).

From (3) we see that $U_{t}$ is the identity on $\Re$ and thus $\left\{U_{t} ;-\infty<t<\infty\right\}$ is a strongly continuous group of unitary operators on all of $\mathcal{H}$. (We no longer restrict the operators in question to R.) The spectral theorem for unitary groups guarantees the existence 
of unique spectral resolution $F\left(\lambda_{2}\right)$ such that

$$
U_{t}=\int_{-\infty}^{\infty} e^{i \lambda_{2} t} d F\left(\lambda_{2}\right), \quad-\infty<t<\infty .
$$

In (e) we saw that $A_{t} U_{s} x=U_{s} A_{t} x$ for all $x \in \tilde{D}$. But for any $x \in D$ we have $x=n+r$ where $n \in \mathcal{N}$ and $r \in \tilde{D}$, hence $A_{t} U_{s} x=U_{s} A_{t} x$ for all $x \in D$ since $\Re$ is the common null space of these operators. For any $x \in D_{1}$ there exists $y_{n} \in D$ such that $y_{n} \rightarrow x$ and $A_{1} y_{n} \rightarrow A_{1} x$. See (c). Hence

$$
\begin{aligned}
U_{t} A_{1} x & =U_{t}\left[\lim _{n \rightarrow \infty} A_{1} y_{n}\right]=\lim _{n \rightarrow \infty} U_{t} A_{1} y_{n} \\
& =\lim _{n \rightarrow \infty} A_{1} U_{t} y_{n} .
\end{aligned}
$$

Moreover $U_{t} y_{n} \rightarrow U_{t} x$ and since $A_{1}$ is closed we have $U_{t} A_{1} x=A_{1} U_{t} x$. Thus $U_{t} A_{1} \subset A_{1} U_{t}$ for $-\infty<t<\infty$. Therefore by Fuglede's theorem [2] we obtain $E\left(\lambda_{1}\right) U_{t}=U_{t} E\left(\lambda_{1}\right)$ for all $t$ and $\lambda_{1}$ and then finally that $E\left(\lambda_{1}\right) F\left(\lambda_{2}\right)=F\left(\lambda_{2}\right) E\left(\lambda_{1}\right)$ for $\lambda_{1}$ and $\lambda_{2}$.

Putting $K(d \lambda)=E\left(d \lambda_{1}\right) F\left(d \lambda_{2}\right)$ we have

$$
N_{t}=A_{t} U_{t}=\int_{\lambda_{1} \geqq_{0}} \lambda_{1}^{i} e^{i \lambda_{2} t} K(d \lambda), \quad t>0 .
$$

Clearly $K(\Lambda)$ is unique on $\mathbb{R}$ but since $\mathscr{N}=K(\{0\}) \mathscr{H}$ it follows that $K(\Lambda)$ is unique on all of $\mathfrak{H C}$.

The author would like to thank the referee for several helpful suggestions which simplified the statement of the theorem.

\section{REFERENCES}

1. A. Devinatz, A note on semi-groups of unbounded self-adjoint operators, Proc. Amer. Math. Soc. vol. 5 (1954) pp. 101-102.

2. B. Fuglede, $A$ commutativity theorem for normal operators, Proc. Nat. Acad. Sci. U.S.A. vol. 36 (1950) pp. 35-41.

3. E. Hille, Functional analysis and semi-groups, Amer. Math. Soc. Colloquium Publications, vol. 31, 1948.

4. B. Sz. Nagy, Spektraldarstellung linearer Transformationen des Hilbertschen Raumes, Berlin, 1942.

Princeton University 